\title{
Inflammatory bowel disease activity assessed by fecal calprotectin and lactoferrin: correlation with laboratory parameters, clinical, endoscopic and histological indexes
}

\author{
Andrea Vieira*1, Chia Bin Fang2, Ernani Geraldo Rolim¹, Wilmar Artur Klug2, \\ Flávio Steinwurz ${ }^{3}$, Lucio Giovanni Battista Rossini ${ }^{4}$ and \\ Paulo Azevedo Candelária²
}

\begin{abstract}
Address: ${ }^{1}$ Clinic of Gastroenterology, Department of Medicine of the Irmandade de Misericórdia da Santa Casa de São Paulo, São Paulo, Brazil ${ }^{2}$ Service of Coloproctology, Department of Surgery of the Irmandade de Misericórdia da Santa Casa de São Paulo, São Paulo, Brazil, ${ }^{3}$ Hospital Israelita Albert Einstein, São Paulo, Brazil and ${ }^{4}$ Service of Endoscopy of Irmandade Santa Casa de São Paulo, São Paulo, Brasil

Email: Andrea Vieira* - andvieira2@uol.com.br; Chia Bin Fang - colon.reto@terra.com.br; Ernani Geraldo Rolim - ernanirolim@uol.com.br; Wilmar Artur Klug - pos.cirurgia@fcmscsp.edu.br; Flávio Steinwurz - flavio@steinwurz.net; Lucio Giovanni

Battista Rossini - luciorossini@uol.com.br; Paulo Azevedo Candelária - paulocandelaria@terra.com.br

* Corresponding author
\end{abstract}

Published: 29 October 2009

BMC Research Notes 2009, 2:22I doi:I0.1 I86/I756-0500-2-22I
Received: 6 July 2009

Accepted: 29 October 2009

This article is available from: http://www.biomedcentral.com/I756-0500/2/22I

(c) 2009 Vieira et al; licensee BioMed Central Ltd.

This is an Open Access article distributed under the terms of the Creative Commons Attribution License (http://creativecommons.org/licenses/by/2.0), which permits unrestricted use, distribution, and reproduction in any medium, provided the original work is properly cited.

\begin{abstract}
Background: Research has shown that fecal biomarkers are useful to assess the activity of inflammatory bowel disease (IBD). The aim of the study is: to evaluate the efficacy of the fecal lactoferrin and calprotectin as indicators of inflammatory activity.

Findings: $A$ total of 78 patients presenting inflammatory bowel disease were evaluated. Blood tests, the Crohn's Disease Activity Index (CDAl), Mayo Disease Activity Index (MDAI), and Crohn's Disease Endoscopic Index of Severity (CDEIS) were used for the clinical and endoscopic evaluation. Two tests were performed on the fecal samples, to check the levels of calprotectin and lactoferrin. The performance of these fecal markers for detection of inflammation with reference to endoscopic and histological inflammatory activity was assessed and calculated sensitivity, specificity, accuracy.

A total of 52 patient's samples whose histological evaluations showed inflammation, 49 were lactoferrin-positive, and 40 were calprotectin-positive $(p=0.000)$. Lactoferrin and calprotectin findings correlated with $C$-reactive protein in both the $C D$ and UC groups $(p=0.006 ; p=0.000)$, with CDAl values $(p=0.043 ; 0.010)$, CDEIS values in DC cases $(p=0,000 ; 0.000)$, and with MDAI values in UC cases $(p=0.000)$.

Conclusion: Fecal lactoferrin and calprotectin are highly sensitive and specific markers for detecting intestinal inflammation. Levels of fecal calprotectin have a proportional correlation to the degree of inflammation of the intestinal mucosa.
\end{abstract}




\section{Findings}

Inflammatory Bowel Disease (IBD) includes Crohn's Disease (CD) and Ulcerative Colitis (UC). These are chronic idiopathic conditions, marked by recurrent episodes of inflammation of the gastrointestinal tract, interspersed with periods of remission. In order to determine the degree of inflammatory activity, it is of the utmost importance to monitor patient's clinical evolution and adjust their therapy [1].

Various indexes are used to evaluate the activity of the disease, which differ from each other in terms of being more subjective (clinical), more objective (endoscopic-histological) or a combination of the two. However, despite the different indexes available, there is not yet any consensus in the literature as to which is the most valid. Laboratory parameters such as C-reactive protein (CRP), erythrocyte sedimentation rate (ESR), and hemoglobin, among others, are not specific to active IBD, which makes it difficult to use them routinely as markers of inflammatory activity in clinical practice [2].

Some authors consider a colonoscopy with biopsy to be the best means for evaluating inflammation location, extent, and severity; aside from being an invasive method, this approach carries risks of complications [3]. Various studies have described fecal markers as powerful biomarkers of inflammation of the intestinal mucosa in patients with IBD [3-8]. Fecal markers selected and studied as indicators of inflammation include neutrophil granule proteins, lactoferrin and calprotectin [2-8].

Lactoferrin is an iron-containing glycoprotein secreted by the majority of mucosal membranes. It is the main component of secondary polymorphonuclear granules, which are the prime cells of an acute inflammatory response. Other hematopoietic cells, such as monocytes and lymphocytes, do not contain lactoferrin. In intestinal inflammation, leukocytes invade the mucosa, which results in an increase in the excretion of lactoferrin into the feces $[5,7]$. Calprotectin is a calcium-containing protein that makes up $5 \%$ of the total protein and $60 \%$ of the cytosolic protein of neutrophil. It has bacteriostatic and fungistatic properties and is found in feces at levels six times higher than that in plasma $[[1,6]$, and [8]].

Several studies have compared fecal lactoferrin and calprotectin with activity indexes and/or endoscopic/histological evaluation to verify intestinal inflammation in IBD patients. The results of these studies are promising, having demonstrated that these markers are useful in detecting inflammation and differentiating it from other diseases as well as in predicting recurrence for periods of up to one year $[[1,2,5,8]$, and [9]]. Hence the present study sought to evaluate the efficacy of fecal excretion biomarkers. Spe- cifically, the first aim was to assess fecal lactoferrin and calprotectin as indicators of IBD activity by determining how well these indicators correlate with other indexes of inflammatory activity including laboratory measures and endoscopic and histological evaluation.

\section{Patients and Methods Patients}

A total of 78 patients, 38 with CD and 40 with UC, were recruited for this study, in accordance with the following criteria for inclusion: (1) age 18 years or older; (2) written informed consent given prior to participation such that the terms were clear and free consent, as approved by the Research Ethics Committee of the Central Hospital of the Santa Casa of São Paulo; (3) being willing and available to undertake all the procedures of the study, such as collection of stool and blood samples and undergoing a colonoscopy and/or double-balloon enteroscopy; and (4) being able to come to the clinic where the study was taking place, whenever necessary. There were five exclusion criteria as follows: (1) testing positive for HIV or Hepatitis $\mathrm{B}$ or $\mathrm{C} ;(2)$ history of infectious diarrhea during the previous six months; (3) infection with intestinal parasites; (4) colostomy or ileostomy up to one month before study enrollment; and (5) prior diagnosis with intestinal cancer. Clinical data for the study's population are summarized in Table 1.

\section{Methods}

\section{The recruitment of patients}

The patients were subjected to blood tests (hemoglobin, hematocrit, leukocytes, platelets, CRP, ESR) and parasitological tests on their feces. The patients selected were advised that they should return to the clinic with two samples of fresh stool collected on the day of or, at most, 24 hours before their visit. In addition, they were asked about

Table I: Summary of IBD patients' clinical data

\begin{tabular}{lcc}
\hline Illness & CD & UC \\
\hline Number of patients & 38 & 40 \\
Sex (male/female) & $24 / 14$ & $21 / 19$ \\
Median age (minimum-maximum) & $37(18-64)$ & $46(19-80)$ \\
Ethnicity (white, asian, mixed-race, black & $32 / 0 / 5 / 1$ & $33 / 1 / 3 / 3$ \\
Family history of IBD (yes/no) & $4 / 34$ & $6 / 34$ \\
Extent of disease & & \\
Terminal ileum & 12 & \\
lleum and colon & 13 & \\
Pancolitis & 11 & 12 \\
Rectal + sigmoid + descending colon & & 3 \\
Rectal & 2 & 25 \\
Surgery intended (Yes/no) & $4 / 34$ & $3 / 37$ \\
Treatment & & \\
Aminosalicylate & 14 & 28 \\
Immunosuppressant & 16 & 11 \\
Anti-TNF & 8 & 1 \\
\hline
\end{tabular}


their symptoms over the preceding seven days in the case of patients with $\mathrm{CD}$, and over the last three days in the case of patients with UC, thereby allowing for the calculation of the Crohn's Disease Activity Index (CDAI) and the Mayo Disease Activity Index (MDAI). CDAI and MDAI values compatible with disease activity were $>150$ points, and $>0$, respectively.

\section{Fecal tests}

During the study, patients' stool samples were subjected to two tests: a quantitative one to determine the level of calprotectin and a qualitative one to identify the presence of lactoferrin. Quantitative measurement of calprotectin was performed using PhiCal ${ }^{\mathrm{TM}}$ Test (Calpro AS, Oslo, Norway). One to $5 \mathrm{~g}$ stool were collected and stored at $-20^{\circ} \mathrm{C}$. Specimens were incubated with polyclonal rabbit antibodies against calprotectin. Bound calprotectin was allowed to reach with alkaline phosphatase labeled, imunoaffinity purified IgG (rabbit) antibodies against calprotectin. After adding enzyme's substrate O.D. values were read in an Elisa reader and compared to negative and positive kit controls. All steps were carried out following manufactures' instructions.

Qualitative measurement of elevated levels of lactoferrin was performed using IBD-CHEK test (Techlab, Blacksburg, VA, USA). One to $5 \mathrm{~g}$ stool were collected. Specimens were incubated with immobilized polyclonal antibody against lactoferrin and then detected with polyclonal antibody conjugated to horseradish peroxidase. After substrate addition, colored enzyme-antibody-antigen complexes formed in the presence of lactoferrin were measured in an Elisa reader and compared to negative and positive kit controls. All steps were carried out following manufactures' instructions.

\section{Endoscopic examinations}

Patients with UC were given a colonoscopy, while those with CD were given a double-balloon enteroscopy (was performed through the rectum) to access inflammatory activity. Two endoscopists (always the same ones) did all of the analyses by video during the procedures without knowledge of the results of the patients' blood tests or fecal tests. Mayo Disease Activity Index (endoscopic sub score), and Crohn's Disease Endoscopic Index of Severity (values $>/=3$ were considered positive for intestinal inflammation) were used for endoscopic evaluation. Biopsies were performed each $10 \mathrm{~cm}$ in normal or pathological areas of bowel.

\section{Histological evaluation}

The following data were assessed by histology: the presence of neutrophils in the lamina propria, the presence of erosion and/or ulceration, and crypt aggression.

\section{Statistical analysis}

The statistical program SPSS (Statistical Package for Social Sciences for Windows version 17.0) was used to analyze the data, with the $5 \%$ level of significance (0.05). Spearman's Rank Correlation was applied to evaluate all the variables studied, namely levels of hemoglobin, hematocrit, leukocytes, platelets, C-reactive protein, hemosedimentation rates, fecal lactoferrin and calprotectin, CDAI, MDAI, CDEIS, and histological evaluation. Sensitivity of the variables was also studied in terms of their positive predictive value, negative predictive value, and accuracy relative to that of endoscopic and histological evaluation.

The best calprotectin cut-off point was calculated using the area under the ROC (receiver operating characteristic) curve.

\section{Results \\ Fecal tests}

Samples were lactoferrin-positive in 49 patients and lactoferrin-negative in 29 patients (Table 2). Mean levels of fecal calprotectin concentration were $686 \mathrm{mg} / \mathrm{kg}$ (ranging from 52,98 to $2542,86 \mathrm{mg} / \mathrm{Kg}$ ). The area under the ROC curve to analyze the cut-off was 0,939 (Figure 1). The best global cut-off was $200,01 \mathrm{mg} / \mathrm{Kg}$ (sensitivity 88,6 ; specificity 97,1$)$. Calprotectin was present at levels above the $200 \mathrm{mg} / \mathrm{kg}$ in 40 patients whose biopsies showed intestinal inflammation (Table 3). As the fecal calprotectin test was quantitative, we chose to evaluate all levels of fecal calprotectin found and to compare them to the degree of inflammation as defined by the histological study of the patients as a whole (Figure 2). All data were analyzed with respect to sensitivity, specificity, positive predictive value,

Table 2: Comparison between fecal lactoferrin assessment and histological evaluation.

\begin{tabular}{|c|c|c|c|c|c|}
\hline \multirow[b]{2}{*}{ Fecal lactoferrin } & \multicolumn{4}{|c|}{ Histological evaluation } & \multirow[b]{2}{*}{ Total } \\
\hline & $\begin{array}{l}\text { Crohn's Disease with } \\
\text { Inflammation }\end{array}$ & $\begin{array}{l}\text { Ulcerative Colitis with } \\
\text { Inflammation }\end{array}$ & $\begin{array}{l}\text { Crohn's Disease } \\
\text { without Inflammation }\end{array}$ & $\begin{array}{l}\text { Ulcerative Colitis } \\
\text { without Inflammation }\end{array}$ & \\
\hline Positive & $22(92 \%)$ & 25 (89.3\%) & $2(14.3 \%)$ & $0(0 \%)$ & $49(62.8 \%)$ \\
\hline Negative & $2(8 \%)$ & $3(10.7 \%)$ & $12(85.7 \%)$ & $12(100 \%)$ & $29(37.2 \%)$ \\
\hline Total & $24(100 \%)$ & $28(100 \%)$ & $14(100 \%)$ & $12(100 \%)$ & $78(100 \%)$ \\
\hline
\end{tabular}

$P=0.000$ (Spearman rank correlation) 


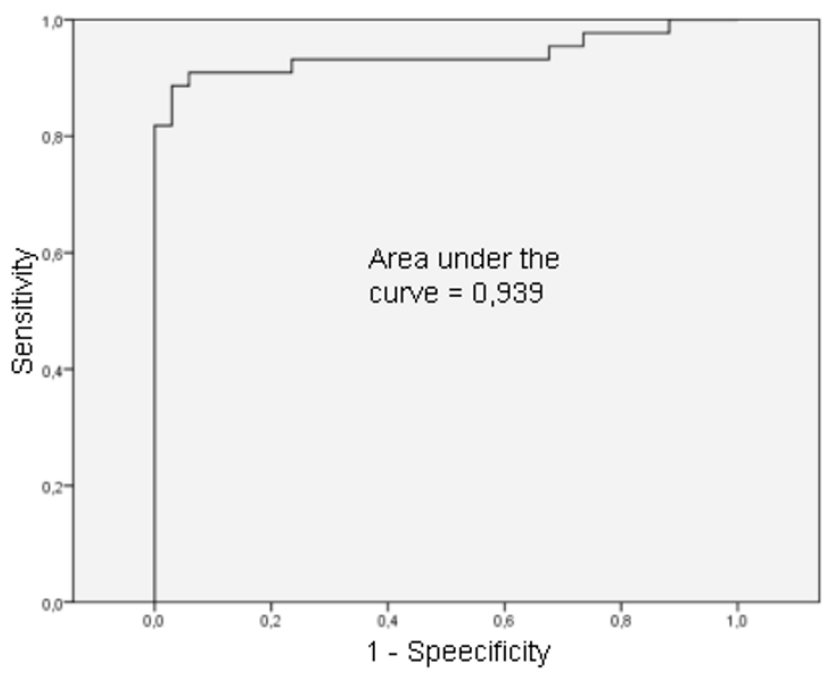

Figure I

Area under the receiver operating characteristic (ROC) curve to analyze the best cut off of calprotectin.

negative predictive value, and accuracy when compared to endoscopic (Table 4) and histological evaluation (Table 5).

\section{Correlation between fecal markers, clinical activity index (CDAI), endoscopy (CDEIS) and clinical-endoscopy (MDAI)}

In patients with $\mathrm{CD}$, lactoferrina and calprotectin results were found to correlate with CDAI ( $p=0,043 ; p=0,010$, respectively) and the CDEIS results $(\mathrm{p}=0.000 ; \mathrm{p}=0.000$, respectively). In patients with $\mathrm{UC}_{\mathrm{C}}$ lactoferrina and calprotectin correlated with MDAI results $(\mathrm{p}=0.000 ; \mathrm{p}=0,000$; respectively).

\section{Correlation between fecal markers and blood tests (C- reactive protein)}

Lactoferrin and calprotectin findings correlated only with C-reactive protein findings in both the CD and UC groups $(\mathrm{p}=0.006 ; \mathrm{p}=0.000)$.

\section{Discussion}

Inflammation is the basis for many signs and symptoms of disease, making its detection and monitoring fundamental to clinical management. At present, a majority of studies have shown that the best way to assess inflammation is through endoscopic and/or histological evaluation [[1,8], and [9]]. Patients' symptoms can be indicators of inflammation and disease activity, but these are subjective and are often influenced by disease factors that are not inflammatory (fibrosis). Various clinical indices developed to calculate inflammatory activity among patients with IBD rely on the combination of signs and symptoms [1].

One means to assess inflammation that has been discussed in recent years is the analysis of the infiltration of neutrophil in the intestinal mucosa and their transmigration to the lumen [10]. When intestinal inflammation occurs, fecal lactoferrin rises rapidly and correlates with endoscopic and histological alterations in patients with IBD, supporting the idea that it is a sensitive and specific means to identity inflammatory activity in these patients [7].

Several authors working on the role of fecal lactoferrin in IBD patients have shown that concentrations of lactoferrin are significantly higher in patients with active disease than in those with inactive disease [7]. These results are consistent with those found in the current study, in which we found that samples from 49 out of 52 patients with intestinal inflammation detected by histological evaluation were positive for lactoferrin.

Another marker derived from neutrophil that has shown great promise for identifying intestinal inflammation is fecal calprotectin [1,11-17]. In patients with active IBD, calprotectin values vary between $200 \mathrm{mg} / \mathrm{kg}$ and 20,000 $\mathrm{mg} / \mathrm{kg}[4,18-21]$.

Many authors have claimed that calprotectin levels correlate closely with histological evaluation than macroscopic findings, suggesting that this biological marker is more sensible than endoscopy in evaluating IBDs activity $[4,8]$. Furthermore fecal calprotectin concentrations predicted the severity of colorectal inflammation, with advanced histological grades of colorectal inflammation [4]. In our study has demonstrated that more intense levels of inflammation are associated with elevated calprotectin values, demonstrating a significant correlation between calprotectin and the severity of inflammation. However the accuracy of calprotectin predicted inflammation when compared with endoscopic and histological were 92, 3\% and $85 \%$.

Calprotectin determination appears to better reflect disease activity in UC than CD [21,22]. As an example, Costa et al. found that fecal calprotectin levels above $50 \mathrm{ug} / \mathrm{g}$ were better correlated with the colitis activity index than the CDAI [21]. The relatively poor correlation between calprotectin levels and CDAI might indeed not be due to a calprotectin pitfall, but to the fact that CDAI is mostly a clinical score and is not sensitive enough to detect subclinical activity of the disease, which is known to occur rather frequently in CD. Nevertheless, several studies could not demonstrate a correlation between fecal calprotectin and 
Table 3: Comparison between fecal calprotectin and histological evaluation.

\begin{tabular}{|c|c|c|c|c|c|}
\hline \multirow[b]{2}{*}{ Fecal calprotectin } & \multicolumn{4}{|c|}{ Histological evaluation } & \multirow[b]{2}{*}{ Total } \\
\hline & $\begin{array}{l}\text { Crohn's Disease with } \\
\text { Inflammation }\end{array}$ & $\begin{array}{l}\text { Ulcerative Colitis } \\
\text { with Inflammation }\end{array}$ & $\begin{array}{c}\text { Crohn's Disease } \\
\text { without } \\
\text { Inflammation }\end{array}$ & $\begin{array}{c}\text { Ulcerative Colitis } \\
\text { without } \\
\text { Inflammation }\end{array}$ & \\
\hline Positive (>200 ng/ml) & $20(83.3 \%)$ & 20 (7I.4\%) & $0(0 \%)$ & $0(0 \%)$ & $40(51.3 \%)$ \\
\hline Negative & $4(16.7 \%)$ & $8(28.6 \%)$ & $14(100 \%)$ & $12(100 \%)$ & $38(48.7 \%)$ \\
\hline Total & $24(100 \%)$ & $28(100 \%)$ & 14 (100\%) & $12(100 \%)$ & $78(100 \%)$ \\
\hline
\end{tabular}

$\mathrm{P}=0.000$ (Spearman rank correlation)

clinical activity of CD (evaluated by CDAI) $[23,24]$ or endoscopic lesions (evaluated by CDEIS) [24].

Sipponen et al. found that both fecal Calprotectin and lactoferrin correlated significantly with CDEIS (Spearman's r 0,729, p < 0,001). With a cut-off level of 200 microg/g for a raised fecal Calprotectin concentration, sensitivity was $70 \%$, specificity $92 \%$ in predicting endoscopically active disease [25].

Other studies have not been able to demonstrate correlation between fecal calprotectin concentration and UCs clinical activity [6]. It has been suggested, but not proved; that CD patients' stratification based on phenotypical pattern (inflammatory, stricturing or fistulizing) could improve calprotectin's predictive capacity for this disease. As calprotectin is an inflammation marker, its predictive role will probably produce best results in the inflammatory pattern of the disease [26]. In summary, the exact strength of any correlation of fecal calprotectin levels with disease activity indicators is therefore not well established at present [27].

In patients with Colitis, it was observed that all patients with clinical and endoscopic signs of inflammation (as determined by the MDAI) had lactoferrin and calprotectin presents in their stool. These findings also occurred in patients with $\mathrm{CD}$; however there were few patients with CDAI higher than 150 points, so further studies are required to resolve this issue.

Both lactoferrin and calprotectin were present in the majority of samples from patients with elevated CRP, but we didn't find a good correlation with fecal markers and

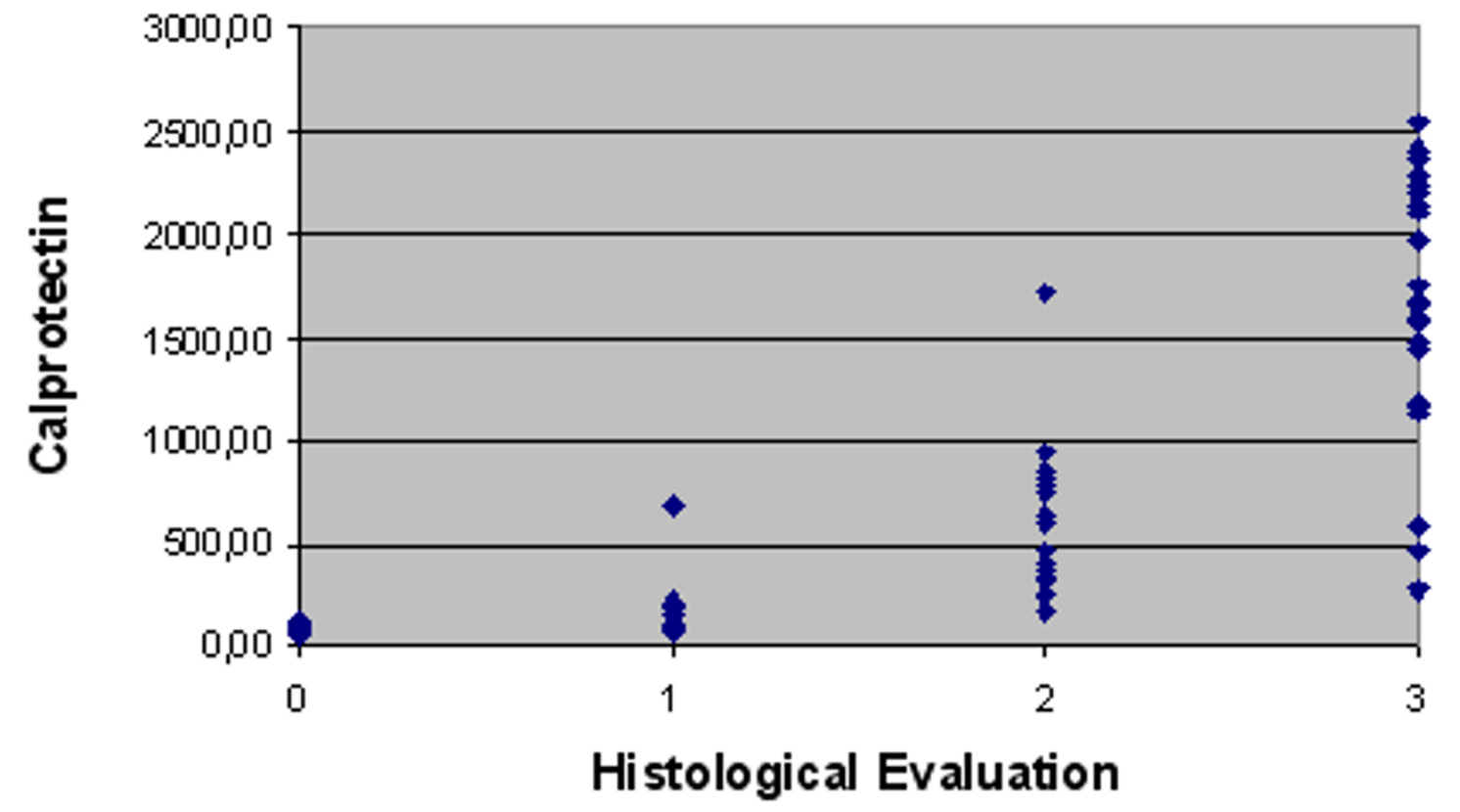

Figure 2

Comparison between fecal Calprotectin and intensity of histological inflammation. $\mathrm{P}=0.000$ (Spearman Rank Correlation). Histological Evaluation (degree of inflammation): 0: absent, I: slight, 2: moderate, 3: severe. 
Table 4: Statistical analysis of activity indexes, blood tests, and fecal markers.

\begin{tabular}{cccccc}
\hline & Sensitivity & Specificity & PPV & NPV & Accuracy \\
\hline CDAl & $26,1 \%$ & $100 \%$ & $100 \%$ & $46,9 \%$ & $55,3 \%$ \\
CRP & $63,6 \%$ & $73,5 \%$ & $75,7 \%$ & $61 \%$ & $67,9 \%$ \\
Lactoferrin & $93,2 \%$ & $76,5 \%$ & $83,7 \%$ & $89,7 \%$ & $85,9 \%$ \\
Calprotectin & $88,6 \%$ & $97,1 \%$ & $97,5 \%$ & $86,8 \%$ & $92,3 \%$ \\
\hline
\end{tabular}

Table 5: Statistical analysis of activity indexes, blood tests, and fecal markers.

\begin{tabular}{cccccc}
\hline & Sensitivity & Specificity & PPV & NPV & Accuracy \\
\hline CDAI & $25 \%$ & $100 \%$ & $100 \%$ & $44 \%$ & $53 \%$ \\
CDEIS & $92 \%$ & $93 \%$ & $96 \%$ & $87 \%$ & $92 \%$ \\
MDAI & $75 \%$ & $100 \%$ & $100 \%$ & $63 \%$ & $83 \%$ \\
CRP & $58 \%$ & $73 \%$ & $81 \%$ & $46 \%$ & $83 \%$ \\
Lactoferrin & $90 \%$ & $92 \%$ & $96 \%$ & $83 \%$ & $91 \%$ \\
Calprotectin & $77 \%$ & $100 \%$ & $100 \%$ & $68 \%$ & $85 \%$ \\
\hline
\end{tabular}

others laboratory parameters. Hemoglobin, hematocrit, leukocytes, platelets and ESR are routinely used as inflammatory markers in blood when IBD is suspected. However, these markers correlate poorly with endoscopic and histological [9], as we also found.

\section{Conclusion}

Several conclusions can be made from the present results. Firstly, fecal lactoferrin and calprotectin are sensitive and specific markers for the detection of intestinal inflammation in IBD patients. Secondly, fecal calprotectin levels are directly proportional to the degree of inflammation in the intestinal mucosa.

\section{Abbreviations}

CDAI: Crohn's Disease Activity Index; MDAI: Mayo Disease ActivityIndex; CDEIS: Crohn's Disease Endoscopic Index of Severity.

\section{Competing interests}

The authors declare that they have no competing interests.

\section{Authors' contributions}

AV performed all manuscript; CBF contributed to manuscript; EGR helped to draft the text; WAK helped to draft the text; LGBR did double-balloon enteroscopy and contributed to manuscript; PAC did colonoscopy and contributed to manuscript. All authors read and approved the final manuscript.

\section{Acknowledgements}

We are grateful to the Support Center for Scientific Publications of the Santa Casa de São Paulo - Faculty of Medical Sciences for their editorial assistance.

\section{References}

I. Konikoff MR, Denson LA: Role of fecal calprotectin as a biomarker of intestinal inflammation in inflammatory bowel disease. Inflamm Bowel Dis 2006, 1 2:524-534.

2. Langhorst J, Elsenbruch S, Mueller T, et al.: Comparison of 4 neutrophil-derived proteins in feces as indicators of disease activity in ulcerative colitis. Inflamm Bowel Dis 2005, I I: |085-109|.

3. Gisbert JP, Mcnicholl AG: Questions and answers on the role of fecal calprotectin as a biological marker in inflammatory bowel disease. Inflamm Bowel Dis 2009, 4 I (I):56-66.

4. Limburg PJ, Ahlquist DA, Sandborn WJ, et al.: Fecal calprotectin levels predict colorectal inflammation among patients with chronic diarrhea referred for colonoscopy. Am J Gastroenterol 2000, 95:283|-2837.

5. Desai D, Faubion WA, Sandborn WJ: Review article: biological activity markers in inflammatory bowel disease. Aliment Pharmacol Ther 2007, 25:247-255.

6. Montalto M, Curigliano V, Santoro L, et al.: Fecal calprotectin in first-degree relatives of patients with ulcerative colitis. Am J Gastroenterol 2007, 102:132-136.

7. Kane SV, Sandborn WJ, Rufo PA, et al:: Fecal lactoferrin is a sensitive and specific marker in identifying intestinal inflammation. Am J Gastroenterol 2003, 98: | 309- 1314.

8. Bunn SK, Bisset WM, Main MJ, et al.: Fecal calprotectin: validation as a noninvasive measure of bowel inflammation in childhood inflammatory bowel disease. J Pediatr Gastroenterol Nutr 2001, 33:14-22

9. Fagerberg UL, Loof L, Myrdal U, et al.: Colorectal inflammation is well predicted by fecal calprotectin in children with gastrointestinal symptoms. J Pediatr Gastroenterol Nutr 2005, 40:450-455.

10. Silberer H, Kuppers B, Mickisch O, et al.: Fecal leukocyte proteins in inflammatory bowel disease and irritable bowel syndrome. Clin Lab 2005, 5 I: I |7-26.

II. Tibble J, Teahon K, Thjodleifsson B, et al: A simple method for assessing intestinal inflammation in Crohn's disease. Gut 2000, 47:506-5I3.

12. Roseth AG, Fagerhol MK, Aadland E, Schjonsby H: Assessment of the neutrophil dominating protein Calprotectin in feces. A methodologic study. Scand J Gastroenterol 1992, 27:793-798.

13. Hanai H, Takeuchi K, Lida T, et al.: Relationship between fecal Calprotectin intestinal inflammation, and peripheral blood neutrophils in patients with active ulcerative colitis. Dig Dis Sci 2004, 49: |438-|443.

14. Tibble JA, Bjarnason I: Fecal Calprotectin as an index of intestinal inflammation. Drugs Today(Barc) 200I, 37:85-96. 
I5. Garcia Sanchez Mdel V, Gonzalez R, Iglesias Flores E, et al.: Diagnostic value of fecal calprotectin in predicting na abnormal colonoscopy. Med Clin (Barc) 2006, I27:4I-46.

16. Berni Canani R, Rapacciuolo L, Romano MT, et al.: Diagnostic value of faecal calprotectin in paediatric gastroenterology clinical practice. Dig Liver Dis 2004, 36:467-470.

17. Roseth AG, Aadland E, Grzyb K: Normalization of faecal Calprotectin: a predictor of mucosal healing in patients with inflammatory bowel disease. Scand ] Gastroenterol 2004, 39: I0 I7-1020.

18. Roseth AG, Aadland E, Jahnsen J, et al:: Assessment of disease activity in ulcerative colitis by faecal calprotectin, a novel granulocyte marker protein. Digestion 1997, 58: I76-180.

19. Roseth AG, Schmidt PN, Fagerhol MK: Correlation between faecal excretion of indium-I I I-labelled granulocytes and calprotectin, a granulocyte marker protein, in patients with inflammatory bowel disease. Scand J Gastroenterol 1999, 34:50-54.

20. Summerton CB, Longlands MG, Wiener K, et al.: Faecal calprotectin: a marker of inflammation throughout the intestinal tract. Eur J Gastroenterol Hepatol 2002, 14:84 I-845.

21. Costa F, Mumolo MG, Bellini M, et al:: Role of faecal calprotectin as non-invasive marker of intestinal inflammation. Dig Liver Dis 2003, 35:642-647.

22. D'Inca R, Dal Pont E, Di Leo, et al.: Calprotectin and lactoferrin in the assessment of intestinal inflammation and organic disease. Int J Colorectal Dis 2007, 22:429-437.

23. Gaya DR, Mackenzie JF: Faecal Calprotectin: a bright future for assessing disease activity in Crohn's disease. QJM 2002, 95:557-558.

24. Denis MA, Reenaers C, Fontaine F, Belaiche J, Louis E: Assessment of endoscopic activity index and biological inflammatory markers in clinically active Crohn's disease with normal Cprotein serum level. Inflamm Bowel Dis 2007, I 3: I I 00-I I 05.

25. Sipponen T, Savilahti E, Kolho KL, Nuutinen H, Turunen U, Farkkila M: Crohn's disease activity assessed by fecal Calprotectin and lactoferrin: correlation with Crohn's disease activity index and endoscopic findings. Inflamm Bowel Dis 2008, I 4(I):40-6.

26. Costa F, Mumolo MG, Ceccarelli L, et al.: Calprotectin is a stronger predictive marker of relapse in ulcerative colitis than in Crohn'sdisease. Gut 2005, 54:364-368.

27. von Roon AC, Karamountzos L, Purkayastha S, et al: Diagnostic precision of fecal Calprotectin for inflammatory bowel disease and colorectal malignancy. Am J Gastroenterol 2007, 1 02:803-8|3.
Publish with Biomed Central and every scientist can read your work free of charge

"BioMed Central will be the most significant development for disseminating the results of biomedical research in our lifetime. "

Sir Paul Nurse, Cancer Research UK

Your research papers will be:

- available free of charge to the entire biomedical community

- peer reviewed and published immediately upon acceptance

- cited in PubMed and archived on PubMed Central

- yours - you keep the copyright
BioMedcentral 